\title{
EMR Study and DFT-Assisted Identification of Transient Radicals in X-Irradiated Crystalline Sucrose
}

Jevgenij Kusakovskij, Freddy Callens and Henk Vrielinck

Department of Solid State Sciences, Ghent University, Krijgslaan 281-S1, 9000 Gent, Belgium

Supporting information 
Table S1. Experimentally determined HF and Schonland conjugate tensors (in MHz) of U1.

\begin{tabular}{|c|c|c|c|c|c|c|}
\hline & & \multirow{2}{*}{$A_{\text {iso }}$} & \multirow{2}{*}{$A_{\text {aniso }}$} & \multicolumn{3}{|c|}{ Eigenvectors } \\
\hline & & & & $a^{*}$ & $b$ & $c$ \\
\hline \multirow{6}{*}{ 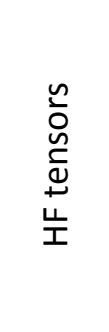 } & & & -29.83 & -0.929 & -0.370 & 0.015 \\
\hline & $\mathrm{H}_{\alpha}$ & -55.51 & -7.66 & 0.254 & -0.665 & -0.702 \\
\hline & & & 37.49 & 0.270 & -0.649 & 0.712 \\
\hline & & & -5.78 & 0.661 & -0.630 & 0.407 \\
\hline & $\mathrm{H}_{\beta}$ & 45.40 & -3.26 & 0.126 & 0.629 & 0.767 \\
\hline & & & 9.04 & -0.739 & -0.456 & 0.495 \\
\hline \multirow{6}{*}{ 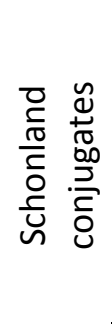 } & & & -34.47 & -0.855 & -0.424 & 0.300 \\
\hline & $\mathrm{H}_{\alpha}$ & -55.67 & 5.22 & -0.516 & 0.628 & -0.583 \\
\hline & & & 29.24 & 0.059 & -0.653 & -0.755 \\
\hline & & & -9.02 & 0.535 & -0.626 & 0.567 \\
\hline & $\mathrm{H}_{\beta}$ & 45.36 & 1.91 & -0.071 & -0.703 & -0.708 \\
\hline & & & 7.11 & -0.842 & -0.338 & 0.420 \\
\hline
\end{tabular}


Figure S1. Figure illustrating the equivalence of wrong rotation sense to erroneous symmetry site assignment in monoclinic systems using the HF tensors from Table S1. The roadmap of the ENDOR line ascribed to the $\mathrm{H}_{\alpha}$ coupling is depicted with solid lines - black for site 1 and red for site 2; the ones ascribed to the Schonland conjugate are depicted with dotted lines - black for site 1 and red for site 2 . For the $\mathrm{H}_{\beta}$ coupling, orange lines were used for site 1 and green - for site 2; the ones ascribed to the Schonland conjugate are depicted with respectively colored dotted lines. Experimental positions of resonance lines of the $\mathrm{H}_{\alpha}$ coupling are presented with filled blue circles and of the $\mathrm{H}_{\beta}$ coupling - purple cirles. Because the rotation planes do not perfectly correspond with bc, ca* and $a * b$, and in particular the degeneracy of the sites in the second plane is lifted, the roadmaps of the Schonland conjugate tensors do not perfectly coincide. As a result, for the $\alpha$-coupling one of the tensors is clearly preferred by the fitting. For the $\beta$-coupling, the preference for one of the tensors is less clear from the fitting, but the Schonland conjugate tensors strongly deviates from the typical b-pattern (see Table S1).

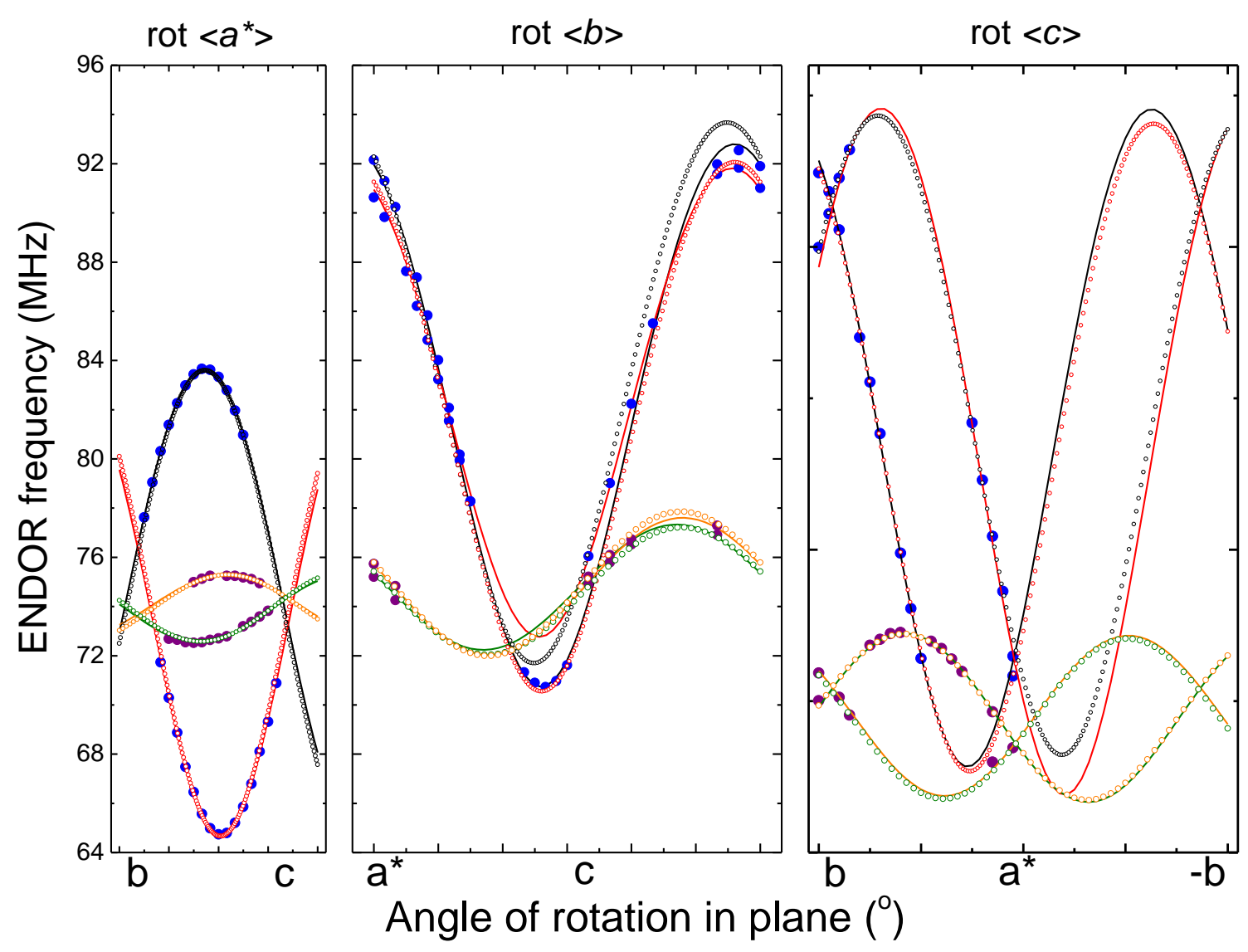

